\title{
Comparison of Prognostic Models of Spontaneous Intracerebral Hemorrhage: Potential Tools for Personalized Care and Clinical Trial in ICH
}

\section{RUIJUN JI}

Beijing Tiantan Hospital

WENJUAN WANG

Beijing Tiantan Hospital

XINYU LIU

Beijing Tiantan Hospital

LINLIN WANG

Beijing Tiantan Hospital

RUIXUAN JIANG

Beijing Tiantan Hospital

RUNHUA ZHANG

Beijing Tiantan Hospital

DANDAN WANG

Beijing Tiantan Hospital

JIAOKUN JIA

Beijing Tiantan Hospital

HAO FENG

Beijing Tiantan Hospital

\section{ZEYU DING}

Beijing Tiantan Hospital

YANFANG LIU

Beijing Tiantan Hospital

\section{GAIFEN LIU}

Beijing Tiantan Hospital

JINGJING LU

Beijing Tiantan Hospital

YI JU

Beijing Tiantan Hospital

XINGQUAN ZHAO ( $\nabla$ zxq@vip.163.com )

Tiantan Comprehensive Stroke Center; Beijing Tiantan Hospital; Capital Medical University; No.119 Nansihuan West Road, Fengtai District, Beijing 100070, China.

\section{Research}

Keywords: Intracerebral hemorrhage, prognostic model, discrimination, calibration, comparison

Posted Date: October 28th, 2020

DOI: https://doi.org/10.21203/rs.3.rs-96623/v1 
License: (9) This work is licensed under a Creative Commons Attribution 4.0 International License. Read Full License 


\section{Abstract}

Background: Several prognostic models have been developed for spontaneous intracerebral hemorrhage (ICH); however, none of them have been consistently used in routine clinical practice or clinical research. In the study, we systematically compared $27 \mathrm{ICH}$ models with regard to mortality and functional outcome at 1-month, 3-month and 1-year after ICH.

Methods: The validation cohort was derived from the Beijing Registration of Intracerebral Hemorrhage. Poor functional outcome was defined as modified Rankin Scale score (mRS) $\geq 3$ at 1-month, 3-month and 1-year after ICH, respectively. The area under the receiver operating characteristic curve (AUROC) and Hosmer-Lemeshow goodness-of-fit test were used to assess model discrimination and calibration. Moderate, good, excellent and outstanding discrimination were predefined as AUROC of $0.75-0.79,0.80-0.84,0.85-0.89$ and above 0.90 , respectively.

Results: A total number of 1575 patients were included. The mean age was $57.2 \pm 14.3$ and $67.2 \%$ were male. The median NIHSS score on admission was 11 (IQR: 3-21). For predicting mortality at 3-month after ICH, AUROC of 27 ICH models ranged from 0.604 to 0.856 . One model showed excellent discrimination; fifteen models demonstrated good discrimination; and seven models demonstrated moderate discrimination. In pairwise comparison, the ICH-FOS $(0.856,95 \% \mathrm{Cl}=0.835-0.878$, $\mathrm{P}<0.001$ ) showed statistically better discrimination than other models for mortality at 3-month after ICH (all $\mathrm{P}<0.05)$. For predicting poor functional outcome (mRS $\geq 3$ ) at 3-month after ICH, AUROC of $27 \mathrm{ICH}$ models ranged from 0.602 to 0.880. Five models showed excellent discrimination; six models demonstrated good discrimination; and ten models demonstrated moderate discrimination. In pairwise comparison with other prediction models, the ICH-FOS was superior in predicting poor functional outcome at 3-month after ICH (all P<0.001). Several risk models were well calibrated (Hosmer-Lemeshow test $\mathrm{P}>0.05$ ) for mortality and poor functional outcome at 3-month after ICH, however, the ICH-FOS showed the largest Cox and Snell R-square. Similar results were verified for mortality and poor functional outcome at 1-month and 1-year after ICH.

Conclusion: Several risk models are externally validated to be effective for risk stratification and outcome prediction after ICH, especially the ICH-FOS, which would be useful tools for personalized care and clinical trial in ICH.

\section{Background}

Spontaneous intracerebral hemorrhage (ICH) accounts for approximately $15-20 \%$ of all strokes and is one of leading causes of mortality and morbidity worldwide[1, 2]. Despite advances in medical knowledge, treatment for ICH remains strictly supportive with not many evidence-based interventions currently available[3, 4]. Effort continues over the development of accurate, reliable and practical clinical grading scales and outcome prediction models for ICH[5], which would be useful for data-driven discussion with patients or families, personalized care and clinical trial.

During the past decades, several prognostic models have been proposed for predicting mortality and functional outcome after ICH. In early stage, ICH models were mainly presented as equations and were not convenient for clinical practice. In 2001, Hemphill et al introduced the original ICH score, which represents as one of the first simple and easily assessable clinical grading scale for $\mathrm{ICH}[6]$. Since then, a number of modifications to the original ICH score and other pragmatic $\mathrm{ICH}$ scores have been developed[5]. Although some of these ICH risk models have been internally or externally validated, none of them has been universally accepted and consistently used in routine clinical practice and clinical research. In addition, with many ICH grading system available, it is becoming increasingly difficult for clinician and researcher to determine which risk models provide optimal predictability and reliability in clinical practice and clinical trials. Therefore, it is necessary to conduct head-to-head comparison of ICH models in an independent cohort.

In the study, we aimed to systematically compare discrimination and calibration of ICH risk models with regard to mortality and functional outcome at 1-month, 3-month and 1-year after ICH following the TRIPOD (transparent reporting of a multivariable prediction model for individual prognosis or diagnosis) guideline[7, 8].

\section{Methods}




\section{Validation cohort}

The validation cohort was derived from the Beijing Registration of Intracerebral Hemorrhage, which was a multicenter, prospective and observational cohort study. A total number of thirteen hospitals in Beijing area participated in the study. Trained research coordinators at each hospital reviewed medical records daily to identify, consent and enroll consecutively eligible patients. To be eligible for the study, subjects had to meet the following criteria: (1) age 18 years or older; (2) hospitalized with a primary diagnosis of spontaneous ICH and confirmed by brain CT or MRI[9]; (3) direct admission to hospital from a physician's clinic or emergency department; (4) written informed consent from patients or their legal representatives. All patients were diagnosed by a certified vascular neurologist. Demographics, medical history, medications, pre-stroke modified Rankin Scale (mRS) score, neurological deficit on admission, blood pressure, laboratory test, neuroimaging, treatment, medical complications and follow-up information during 1-year after onset were prospectively recorded. The study protocol was approved by the Institutional Review Board (IRB) of the Beijing Tiantan Hospital (KY2014023-02) and written informed consent was obtained.

\section{Data Collection And Definition Of Variables}

A standardized electronic case report form (eCRF) was used for data collection. Participating centers collected data and submitted it online to the coordinating center at Beijing Tiantan Hospital. For this study, the following candidate variables were analyzed: (1) demographics (age and gender); (2) time from onset to hospital; (3) stroke risk factors: hypertension (history of hypertension or anti-hypertensive medication use), diabetes mellitus (history of diabetes mellitus or anti-diabetic medication use), dyslipidemia (history of dyslipidemia or lipid-lowering medication use), atrial fibrillation (history of atrial fibrillation or documentation of atrial fibrillation on admission), history of stroke/TIA, myocardial infarction, heart failure, current smoking, and heavy alcohol consumption ( $\geq 2$ standard alcohol beverage per day); (4) pre-admission antithrombotic medications (anticoagulation and antiplatelet agents); (5) pre-stroke mRS score; (6) admission stroke severity based on the National Institutes of Health Stroke Scale score (NIHSS) and the Glasgow Coma Scale (GCS) score; (7) admission systolic and diastolic blood pressure ( $\mathrm{mmHg}$ ): (8) admission laboratory tests (white blood cell count, blood glucose, and creatinine); (9) neuroimaging variables: intracerebral hemorrhage volume (measured using the $A B C / 2$ method[10]), hematoma location (supratentorial or infratentorial ICH), intraventricular extension (presence or absence) and subarachnoid extension (presence or absence). All images were prospectively viewed by a trained neuroradiologist blinded to clinical data. (10) etiology diagnosis at discharge (primary or secondary ICH); (11) surgical treatment (craniotomy evacuation, minimal-invasive surgical therapy or brain ventricle puncture and drainage); (12) withdrawal of medical care; and (13) length of hospital stay (LOS).

\section{Functional Outcome Assessment}

The modified Rankin Scale was used to evaluate functional outcome during 1 year after ICH. Central follow-up blinded to baseline variables was made by telephone interview by trained interviewers based on a standardized interview protocol. For patients who lost to follow-up, we made telephone follow-up interview once a week for three times. In the study, poor functional outcome was defined as $\mathrm{mRS} \geq 3$ at 1-month, 3-month and 1-year after ICH.

\section{Statistical analysis}

Continuous variables were summarized with mean and standard deviation (SD) or median and interquartile range (IQR). Categorical variables were summarized as proportions. Chi-square or Fisher exact test was used to compare categorical variables and Mann-Whitney test or independent t-test was employed to compare continuous variables between groups.

The primary criterion for selecting ICH model was whether all elements required for the model was available in our dataset and finally 27 existing ICH risk models met this criterion: the Tuhrim's Eq. (1991)[11], Broderick's score (1993)[12], Lisk's Eq. (1994)[13], Mase's Eq. (1995)[14], original ICH Score (2001)[6], Hallevy's score (2002)[15], modified ICH Score (2003)[16], 
Shaya's score (2005)[17], Essen ICH score (2006)[18], Weimar' equation-I (2006)[19], GP on STAGE score (2006)[20], ICH-GS (2007)[21], FUNC Score (2008)[22], MICH Score (2008)[23], Huang's score (2008)[24], simplified ICH score (2009)[25], Weimar' equation-II (2009)[26], IVH score (2010)[27], Kinshasa ICH score (2012)[28], ICH index (2012)[29], ICH-FOS (2013)[30], EDICH score (2014)[31], CAA-ICH score (2016)[32], Ho's score (2016)[33], Safatli's score (2016)[34], max-ICH score (2017)[35] and ICHOP score (2017)[36].

The resulting $\mathrm{ICH}$ models were validated by assessing model discrimination and calibration[7, 8]. Discrimination was assessed by calculating the area under the receiver operating characteristic curve (AUROC). In the study, moderate, good, excellent and outstanding discrimination were predefined as AUROC of $0.75-0.79,0.80-0.84,0.85-0.89$ and above 0.90 , respectively. Due to that existing $\mathrm{ICH}$ models were designed for predicting outcomes at different time points after onset, we compared discrimination of $\mathrm{ICH}$ models with regard to mortality and poor functional outcome at 1-month, 3-month and 1-year after ICH, respectively. Pairwise AUROC was compared by using Delong's method[37] and sensitivity, specificity, positive predict value (PPV), and negative predictive value (NPV) were calculated at each risk models' maximum Youden Index. Calibration was assessed by performing the Hosmer-Lemeshow goodness-of-fit test and plot of observed versus predicted risk according to 10 deciles of the predicted risk. The Cox and Snell R-square and Nagelkerke R-square of the HosmerLemeshow goodness-of-fit test were calculated.

All tests were 2-tailed and statistical significance was determined at a level of 0.05 . Statistical analysis was performed using SAS 9.1 (SAS Institute, Cary, NC), SPSS 19.0 (SPSS Inc., Chicago, IL), and Medcalc software 12.3 (MedCalc).

\section{Results}

\section{Patient characteristics}

From December 2014 to September 2016, a total number of 1964 patients were enrolled in the Beijing Registration of Intracerebral Hemorrhage. Among them, we were unable to obtain follow-up information in 389, yielding 1575 patients with requisite data for the present analysis (An additional figure shows this in more detail [see Additional Fig. 1]). The clinical characteristics of the study patients are shown in Table 1. The mean age was $57.2 \pm 14.3$ and $67.2 \%$ were male. The median time from onset to hospital was 4.0 hours (IQR: 1.83-11.1). The median NIHSS and GCS score on admission was 11 (IQR: 3$21)$ and 14 (IQR: 8-15), respectively. A total number of 1438 (91.3) was diagnosed with primary ICH and 137 (8.7\%) secondary ICH at discharge. The median length of hospital stay was 16 days (IQR: 8-22). There were 104 (6.6\%) patients in whom withdraw of care was instituted during hospitalization. The median mRS score at 1-month, 3-month and 1-year after onset was 4 (IQR: 1-5), 3 (IQR: 1-5) and 2 (IQR: 1-6), respectively. The baseline characteristics of patients included $(n=$ $1575)$ and those excluded $(n=389)$ are listed in additional Table 1 (An additional table shows this in more detail [see Additional Table 1]). They were not statistically different in initial NIHSS score, GCS score, hematoma volume and withdraw of care. 


\begin{tabular}{|c|c|}
\hline & $\begin{array}{l}\text { Overall } \\
(n=1575)\end{array}$ \\
\hline \multicolumn{2}{|l|}{ Demographics } \\
\hline Age, y, median (IQR) & $57.2 \pm 14.3$ \\
\hline Gender (male), n (\%) & $1061(67.2)$ \\
\hline Onset to hospital (hours), median (IQR) & $4.00(1.83-11.1)$ \\
\hline \multicolumn{2}{|l|}{ Risk factors, n (\%) } \\
\hline Hypertension & 1093 (69.4) \\
\hline Diabetes mellitus & $236(15.0)$ \\
\hline Dyslipidemia & $150(9.5)$ \\
\hline Atrial fibrillation & $23(1.5)$ \\
\hline History of stroke/TIA & $263(16.7)$ \\
\hline Myocardial infarction & $35(2.2)$ \\
\hline Heart failure & $7(0.4)$ \\
\hline Current smoker & $511(32.4)$ \\
\hline Heavy alcohol consumption & $589(37.4)$ \\
\hline Pre-admission anticoagulation, n (\%) & $17(1.1)$ \\
\hline Pre-admission antiplatelet, n (\%) & $246(15.6)$ \\
\hline Pre-stroke mRS score, median (IQR) & $0(0-0)$ \\
\hline Admission NIHSS score, median (IQR) & $11(3-21)$ \\
\hline Admission GCS score, median (IQR) & $14(8-15)$ \\
\hline Admission SBP (mm Hg), median (IQR) & $164(147-187)$ \\
\hline Admission DBP (mm Hg), median (IQR) & $95(82-109)$ \\
\hline Admission WBC, $10^{9} / \mathrm{L}$, median (IQR) & $9.83(7.41-13.1)$ \\
\hline Admission glucose (mmol/L), median (IQR) & $7.4(6.1-9.3)$ \\
\hline Admission creatinine (mmol/L), median (IQR) & $63.8(52.7-77.2)$ \\
\hline \multicolumn{2}{|l|}{ Hematoma location } \\
\hline Supratentorial ICH,n (\%) & $1384(87.9)$ \\
\hline Infratentorial ICH, n (\%) & $191(12.1)$ \\
\hline Hematoma volume $\left(\mathrm{cm}^{3}\right)$, median (IQR) & $16.1(6.2-39.5)$ \\
\hline Intraventricular extension, n (\%) & $181(11.5)$ \\
\hline
\end{tabular}

Abbreviation: IQR, Interquartile Range; TIA, Transient Ischemic Attack; NIHSS, National Institutes of Health Stroke Scale score; GCS, Glasgow Coma Scale; SBP, Systolic Blood Pressure; DBP, Diastolic Blood Pressure; WBC, White Cell Count; ICH, Intracerebral Hemorrhage; mRS, modified Rankin Scale. 


\begin{tabular}{|c|c|}
\hline & $\begin{array}{l}\text { Overall } \\
(n=1575)\end{array}$ \\
\hline Subarachnoid extension, n (\%) & $230(14.6)$ \\
\hline \multicolumn{2}{|l|}{ Etiology diagnosis } \\
\hline Primary $\mathrm{ICH}$ & $1438(91.3)$ \\
\hline Secondary $\mathrm{ICH}$ & $137(8.7)$ \\
\hline Withdrawal of medical care, $\mathrm{n}(\%)$ & $104(6.6)$ \\
\hline Surgical treatment & $312(19.8)$ \\
\hline Length of hospital stay, median (IQR) & $16(8-22)$ \\
\hline \multicolumn{2}{|l|}{ Outcomes within 1-year after onset } \\
\hline mRS score at 1-month after onset, median (IQR) & $4(1-5)$ \\
\hline mortality at 1-month after onset, $\mathrm{n}(\%)$ & $313(19.9)$ \\
\hline $\mathrm{mRS} \geq 3$ at 1 -month after onset, $\mathrm{n}(\%)$ & $966(61.3)$ \\
\hline mRS score at 3-month after onset, median (IQR) & $3(1-5)$ \\
\hline mortality at 3-month after onset, n (\%) & $337(21.4)$ \\
\hline mRS $\geq 3$ at 3-month after onset, $\mathrm{n}(\%)$ & $869(55.2)$ \\
\hline mRS score at 1-year after onset, median (IQR) & $2(1-6)$ \\
\hline mortality at 1-year after onset, n (\%) & $399(25.3)$ \\
\hline mRS $\geq 3$ at 1 -year after onset, $n(\%)$ & $774(49.1)$ \\
\hline
\end{tabular}

\section{Predictive Performance For Mortality After Ich}

Discrimination. Figure 1 shows discrimination of $27 \mathrm{ICH}$ models with regard to mortality and poor functional outcome (mRS $\geq 3$ ) at 1-month, 3-month and 1-year after ICH, respectively. For predicting 3-month mortality after ICH, AUROC of $27 \mathrm{ICH}$ risk models ranged from 0.604 to 0.856 . Among them, one model (The ICH-FOS) showed excellent discrimination with AUROC between 0.85 and 0.89 ; fifteen risk models demonstrated good discrimination with AUROC between 0.80 and 0.84 including the Essen ICH score, Weimar' equation-II, GP on STAGE score, EDICH score, modified ICH Score, Huang's score, ICHOP score, Lisk's equation, CAA-ICH score, FUNC Score, simplified ICH score, ICH index, IVH score, max-ICH score and Mase's equation; and seven risk models demonstrated moderate discrimination with AUROC between 0.75 and 0.79 (Table 2) (An additional figure shows this in more detail [see Additional Fig. 2]). The sensitivity, specificity, PPV, NPV and maximum Youden Index of $27 \mathrm{ICH}$ models for predicting 3-month mortality after ICH are shown in Table 2. The ICH-FOS showed the maximum Youden Index. In pairwise comparison, the ICH-FOS $(0.856,95 \% \mathrm{Cl}=0.835-0.878, \mathrm{P}<0.001)$ showed statistically better discrimination than other risk models for mortality at 3-month after ICH (all $\mathrm{P}<0.05$ ). Similar results were found with regard to mortality at 1 month and 1-year after ICH (Additional tables show this in more detail [see Additional Table 2-3]). 
Table 2

Discrimination of $\mathrm{ICH}$ risk models with regard to mortality and poor functional outcome $(\mathrm{mRS} \geq 3)$ at 3-month after ICH $(\mathrm{n}=$ 1575)

\begin{tabular}{|c|c|c|c|c|c|c|c|c|c|c|}
\hline & AUROC & $95 \% \mathrm{Cl}$ & $\stackrel{\Delta}{A U R O C *}$ & $\begin{array}{l}\text { P } \\
\text { value \& }\end{array}$ & $\begin{array}{l}\text { Youden } \\
\text { Index }\end{array}$ & Cutoff & Sensitivity & Specificity & PPV & NPV \\
\hline \multicolumn{11}{|l|}{$\begin{array}{l}\text { Mortality } \\
\text { at 3- } \\
\text { month } \\
\text { after ICH }\end{array}$} \\
\hline $\begin{array}{l}\text { The ICH- } \\
\text { FOS } \\
(2013)\end{array}$ & 0.856 & $\begin{array}{l}0.835- \\
0.878\end{array}$ & Reference & & 0.577 & 8 & 0.798 & 0.779 & 0.500 & 0.934 \\
\hline $\begin{array}{l}\text { The Essen } \\
\text { ICH score } \\
(2006)\end{array}$ & 0.839 & $\begin{array}{l}0.820- \\
0.857\end{array}$ & 0.017 & 0.02 & 0.566 & 4 & 0.777 & 0.788 & 0.501 & 0.929 \\
\hline $\begin{array}{l}\text { Weimar' } \\
\text { equation-II } \\
\text { (2009) }\end{array}$ & 0.836 & $\begin{array}{l}0.817- \\
0.854\end{array}$ & 0.020 & 0.03 & 0.561 & $\ldots$ & 0.756 & 0.805 & 0.514 & 0.924 \\
\hline $\begin{array}{l}\text { The GP on } \\
\text { STAGE } \\
\text { score } \\
(2006)\end{array}$ & 0.836 & $\begin{array}{l}0.816- \\
0.854\end{array}$ & 0.020 & 0.03 & 0.571 & $\ldots$ & 0.750 & 0.821 & 0.534 & 0.924 \\
\hline $\begin{array}{l}\text { The EDICH } \\
\text { score } \\
\text { (2014) }\end{array}$ & 0.836 & $\begin{array}{l}0.817- \\
0.854\end{array}$ & 0.020 & 0.04 & 0.567 & 2 & 0.730 & 0.837 & 0.550 & 0.919 \\
\hline $\begin{array}{l}\text { The } \\
\text { modified } \\
\text { ICH Score } \\
(2003)\end{array}$ & 0.835 & $\begin{array}{l}0.815- \\
0.853\end{array}$ & 0.021 & $<0.001$ & 0.551 & 2 & 0.765 & 0.785 & 0.493 & 0.925 \\
\hline $\begin{array}{l}\text { Huang's } \\
\text { score } \\
\text { (2008) }\end{array}$ & 0.829 & $\begin{array}{l}0.809- \\
0.847\end{array}$ & 0.027 & $\begin{array}{l}< \\
0.001\end{array}$ & 0.571 & 3 & 0.715 & 0.856 & 0.575 & 0.917 \\
\hline $\begin{array}{l}\text { ICHOP } \\
(2018)\end{array}$ & 0.828 & $\begin{array}{l}0.809- \\
0.847\end{array}$ & 0.028 & $\begin{array}{l}<.001 \\
0.001\end{array}$ & 0.557 & 5 & 0.744 & 0.812 & 0.520 & 0.921 \\
\hline $\begin{array}{l}\text { Lisk's } \\
\text { Eq. (1994) }\end{array}$ & 0.827 & $\begin{array}{l}0.807- \\
0.845\end{array}$ & 0.029 & $\begin{array}{l}< \\
0.001\end{array}$ & 0.553 & $\ldots$ & 0.727 & 0.826 & 0.533 & 0.917 \\
\hline $\begin{array}{l}\text { CAA- ICH } \\
\text { score } \\
(2016)\end{array}$ & 0.827 & $\begin{array}{l}0.807- \\
0.845\end{array}$ & 0.029 & $\begin{array}{l}< \\
0.001\end{array}$ & 0.519 & 1 & 0.872 & 0.647 & 0.403 & 0.949 \\
\hline $\begin{array}{l}\text { The FUNC } \\
\text { Score } \\
(2008)\end{array}$ & 0.825 & $\begin{array}{l}0.805- \\
0.843\end{array}$ & 0.031 & $\begin{array}{l}<.001 \\
0.01\end{array}$ & 0.538 & 7 & 0.845 & 0.692 & 0.429 & 0.943 \\
\hline $\begin{array}{l}\text { The } \\
\text { simplified } \\
\text { ICH score } \\
(2009)\end{array}$ & 0.825 & $\begin{array}{l}0.805- \\
0.844\end{array}$ & 0.031 & $\begin{array}{l}< \\
0.001\end{array}$ & 0.508 & 7 & 0.649 & 0.858 & 0.556 & 0.900 \\
\hline
\end{tabular}

* $\triangle$ AUROC denotes the difference in AUROC between the ICH-FOS and compared scores with regard to mortality and poor functional outcome at 3-month after $\mathrm{ICH}$.

\& $\mathrm{P}$ value of comparing paired AUROC with Delong's method.

Abbreviation: ICH, Intracerebral Hemorrhage; AUROC, Area Under the Receiver Operating Characteristic Curve; $\mathrm{Cl}$, Confidence Interval; PPV, Positive Predictive Value; NPV, Negative Predictive Value. 


\begin{tabular}{|c|c|c|c|c|c|c|c|c|c|c|}
\hline & AUROC & $95 \% \mathrm{Cl}$ & $\stackrel{\Delta}{\text { AUROC* }}$ & $\begin{array}{l}\text { P } \\
\text { value }^{\&}\end{array}$ & $\begin{array}{l}\text { Youden } \\
\text { Index }\end{array}$ & Cutoff & Sensitivity & Specificity & PPV & NPV \\
\hline $\begin{array}{l}\text { The ICH } \\
\text { index } \\
(2012)\end{array}$ & 0.821 & $\begin{array}{l}0.801- \\
0.840\end{array}$ & 0.035 & $\begin{array}{l}< \\
0.001\end{array}$ & 0.530 & $\ldots$ & 0.747 & 0.782 & 0.484 & 0.919 \\
\hline $\begin{array}{l}\text { The IVH } \\
\text { score } \\
\text { (2010) }\end{array}$ & 0.818 & $\begin{array}{l}0.798- \\
0.837\end{array}$ & 0.038 & $\begin{array}{l}< \\
0.001\end{array}$ & 0.555 & 1 & 0.836 & 0.718 & 0.448 & 0.942 \\
\hline $\begin{array}{l}\max -\mathrm{ICH} H \\
(2017)\end{array}$ & 0.813 & $\begin{array}{l}0.793- \\
0.832\end{array}$ & 0.043 & $\begin{array}{l}< \\
0.001\end{array}$ & 0.519 & 2 & 0.854 & 0.665 & 0.410 & 0.944 \\
\hline $\begin{array}{l}\text { Mase's } \\
\text { Eq. (1995) }\end{array}$ & 0.809 & $\begin{array}{l}0.788- \\
0.828\end{array}$ & 0.047 & $\begin{array}{l}< \\
0.001\end{array}$ & 0.512 & $\ldots$ & 0.798 & 0.714 & 0.432 & 0.928 \\
\hline $\begin{array}{l}\text { The } \\
\text { original } \\
\text { ICH Score } \\
(2001)\end{array}$ & 0.797 & $\begin{array}{l}0.777- \\
0.817\end{array}$ & 0.059 & $<0.001$ & 0.475 & 1 & 0.768 & 0.707 & 0.417 & 0.918 \\
\hline $\begin{array}{l}\text { The ICH- } \\
\text { GS (2007) }\end{array}$ & 0.797 & $\begin{array}{l}0.777- \\
0.817\end{array}$ & 0.059 & $<.001$ & 0.475 & 1 & 0.768 & 0.707 & 0.417 & 0.918 \\
\hline $\begin{array}{l}\text { The MICH } \\
\text { Score } \\
\text { (2008) }\end{array}$ & 0.794 & $\begin{array}{l}0.774- \\
0.814\end{array}$ & 0.062 & $\begin{array}{l}<.001 \\
0.00\end{array}$ & 0.465 & 1 & 0.768 & 0.696 & 0.409 & 0.917 \\
\hline $\begin{array}{l}\text { Broderick's } \\
\text { score } \\
(1993)\end{array}$ & 0.776 & $\begin{array}{l}0.754- \\
0.797\end{array}$ & 0.080 & $\begin{array}{l}< \\
0.001\end{array}$ & 0.521 & $\ldots$ & 0.835 & 0.686 & 0.394 & 0.945 \\
\hline $\begin{array}{l}\text { Ho's score } \\
(2016)\end{array}$ & 0.772 & $\begin{array}{l}0.751- \\
0.793\end{array}$ & 0.084 & $<0.001$ & 0.476 & $\ldots$ & 0.774 & 0.702 & 0.415 & 0.919 \\
\hline $\begin{array}{l}\text { Hallevy's } \\
\text { score } \\
(2002)\end{array}$ & 0.770 & $\begin{array}{l}0.748- \\
0.790\end{array}$ & 0.086 & $\begin{array}{l}< \\
0.001\end{array}$ & 0.402 & 2 & 0.685 & 0.716 & 0.397 & 0.893 \\
\hline $\begin{array}{l}\text { Tuhrim's } \\
\text { Eq. (1991) }\end{array}$ & 0.761 & $\begin{array}{l}0.739- \\
0.782\end{array}$ & 0.095 & $\begin{array}{l}<.001 \\
0.00\end{array}$ & 0.441 & $\ldots$ & 0.655 & 0.785 & 0.455 & 0.893 \\
\hline $\begin{array}{l}\text { The } \\
\text { Kinshasa } \\
\text { ICH score } \\
(2012)\end{array}$ & 0.683 & $\begin{array}{l}0.659- \\
0.706\end{array}$ & 0.173 & $\dot{0} 001$ & 0.320 & 6 & 0.646 & 0.673 & 0.350 & 0.875 \\
\hline $\begin{array}{l}\text { Shaya's } \\
\text { score } \\
(2005)\end{array}$ & 0.636 & $\begin{array}{l}0.611- \\
0.659\end{array}$ & 0.220 & $<.001$ & 0.247 & 2 & 0.599 & 0.647 & 0.317 & 0.856 \\
\hline $\begin{array}{l}\text { Weimar' } \\
\text { equation-I } \\
\text { (2006) }\end{array}$ & 0.632 & $\begin{array}{l}0.607- \\
0.655\end{array}$ & 0.224 & $\begin{array}{l}< \\
0.001\end{array}$ & 0.192 & $\ldots$ & 0.549 & 0.643 & 0.296 & 0.839 \\
\hline $\begin{array}{l}\text { Safatli's } \\
\text { score } \\
(2016)\end{array}$ & 0.604 & $\begin{array}{l}0.579- \\
0.628\end{array}$ & 0.252 & $\begin{array}{l}< \\
0.001\end{array}$ & 0.207 & 0 & 0.299 & 0.907 & 0.470 & 0.826 \\
\hline \multicolumn{11}{|c|}{ Poor functional outcome (mRS $\geq 3$ ) at 3-month after ICH } \\
\hline \multicolumn{11}{|c|}{$\begin{array}{l}\text { * } \triangle \text { AUROC denotes the difference in AUROC between the ICH-FOS and compared scores with regard to mortality and poor } \\
\text { functional outcome at 3-month after ICH. }\end{array}$} \\
\hline \multicolumn{11}{|c|}{ \& $P$ value of comparing paired AUROC with Delong's method. } \\
\hline $\begin{array}{l}\text { Abbreviatic } \\
\text { Confidence }\end{array}$ & $\mathrm{H}$, Intr & ebral 1 & rrhage & C, A & nder $t$ & ceiv & erating $\mathrm{C}$ & teristic $C$ & $\mathrm{Cl}$ & \\
\hline
\end{tabular}




\begin{tabular}{|c|c|c|c|c|c|c|c|c|c|c|}
\hline & AUROC & $95 \% \mathrm{Cl}$ & $\stackrel{\Delta}{A}$ AUROC* & $\begin{array}{l}\text { P } \\
\text { value }^{\&}\end{array}$ & $\begin{array}{l}\text { Youden } \\
\text { Index }\end{array}$ & Cutoff & Sensitivity & Specificity & PPV & NPV \\
\hline $\begin{array}{l}\text { The ICH- } \\
\text { FOS } \\
(2013)\end{array}$ & 0.880 & $\begin{array}{l}0.863- \\
0.896\end{array}$ & Reference & & 0.588 & 4 & 0.756 & 0.832 & 0.848 & 0.734 \\
\hline $\begin{array}{l}\text { The Essen } \\
\text { ICH score } \\
(2006)\end{array}$ & 0.862 & $\begin{array}{l}0.843- \\
0.878\end{array}$ & 0.018 & $\begin{array}{l}<.001 \\
0.001\end{array}$ & 0.554 & 2 & 0.784 & 0.769 & 0.808 & 0.743 \\
\hline $\begin{array}{l}\max -\mathrm{ICH} H \\
(2017)\end{array}$ & 0.862 & $\begin{array}{l}0.844- \\
0.878\end{array}$ & 0.018 & $\begin{array}{l}<.001 \\
0.00\end{array}$ & 0.562 & 1 & 0.872 & 0.690 & 0.777 & 0.814 \\
\hline $\begin{array}{l}\text { Weimar' } \\
\text { equation-II } \\
\text { (2009) }\end{array}$ & 0.853 & $\begin{array}{l}0.835- \\
0.870\end{array}$ & 0.027 & $\begin{array}{l}< \\
0.001\end{array}$ & 0.535 & $\ldots$ & 0.848 & 0.687 & 0.770 & 0.786 \\
\hline $\begin{array}{l}\text { ICHOP } \\
(2018)\end{array}$ & 0.850 & $\begin{array}{l}0.832- \\
0.867\end{array}$ & 0.030 & $\begin{array}{l}<.001 \\
0.00\end{array}$ & 0.533 & 3 & 0.735 & 0.798 & 0.818 & 0.710 \\
\hline $\begin{array}{l}\text { Hallevy's } \\
\text { score } \\
(2002)\end{array}$ & 0.840 & $\begin{array}{l}0.821- \\
0.858\end{array}$ & 0.040 & $\begin{array}{l}< \\
0.001\end{array}$ & 0.550 & 1 & 0.850 & 0.703 & 0.778 & 0.791 \\
\hline $\begin{array}{l}\text { The } \\
\text { modified } \\
\text { ICH Score } \\
(2003)\end{array}$ & 0.818 & $\begin{array}{l}0.798- \\
0.837\end{array}$ & 0.062 & $\begin{array}{l}< \\
0.001\end{array}$ & 0.489 & 1 & 0.716 & 0.772 & 0.796 & 0.689 \\
\hline $\begin{array}{l}\text { Lisk's } \\
\text { Eq. (1994) }\end{array}$ & 0.815 & $\begin{array}{l}0.795- \\
0.834\end{array}$ & 0.065 & $\begin{array}{l}<.001 \\
0.00\end{array}$ & 0.485 & $\ldots$ & 0.558 & 0.927 & 0.905 & 0.630 \\
\hline $\begin{array}{l}\text { The GP on } \\
\text { STAGE } \\
\text { score } \\
(2006)\end{array}$ & 0.811 & $\begin{array}{l}0.791- \\
0.830\end{array}$ & 0.069 & $\begin{array}{l}< \\
0.001\end{array}$ & 0.494 & $\ldots$ & 0.680 & 0.813 & 0.819 & 0.673 \\
\hline $\begin{array}{l}\text { Mase's } \\
\text { Eq. (1995) }\end{array}$ & 0.807 & $\begin{array}{l}0.787- \\
0.827\end{array}$ & 0.073 & $\begin{array}{l}< \\
0.001\end{array}$ & 0.478 & $\ldots$ & 0.631 & 0.848 & 0.837 & 0.650 \\
\hline $\begin{array}{l}\text { The IVH } \\
\text { score } \\
(2010)\end{array}$ & 0.806 & $\begin{array}{l}0.786- \\
0.825\end{array}$ & 0.074 & $\begin{array}{l}< \\
0.001\end{array}$ & 0.501 & 1 & 0.624 & 0.876 & 0.862 & 0.654 \\
\hline $\begin{array}{l}\text { The FUNC } \\
\text { Score } \\
(2008)\end{array}$ & 0.797 & $\begin{array}{l}0.776- \\
0.817\end{array}$ & 0.083 & $\begin{array}{l}< \\
0.001\end{array}$ & 0.495 & 7 & 0.644 & 0.850 & 0.842 & 0.660 \\
\hline $\begin{array}{l}\text { The MICH } \\
\text { Score } \\
\text { (2008) }\end{array}$ & 0.792 & $\begin{array}{l}0.771- \\
0.811\end{array}$ & 0.088 & $\begin{array}{l}< \\
0.001\end{array}$ & 0.451 & 1 & 0.605 & 0.846 & 0.830 & 0.635 \\
\hline $\begin{array}{l}\text { CAA-ICH } \\
\text { score } \\
(2016)\end{array}$ & 0.791 & $\begin{array}{l}0.770- \\
0.811\end{array}$ & 0.089 & $\begin{array}{l}< \\
0.001\end{array}$ & 0.495 & 1 & 0.685 & 0.809 & 0.816 & 0.676 \\
\hline $\begin{array}{l}\text { The ICH } \\
\text { index } \\
(2012)\end{array}$ & 0.787 & $\begin{array}{l}0.766- \\
0.807\end{array}$ & 0.093 & $\begin{array}{l}< \\
0.001\end{array}$ & 0.450 & $\ldots$ & 0.676 & 0.774 & 0.787 & 0.660 \\
\hline
\end{tabular}

* $\triangle$ AUROC denotes the difference in AUROC between the ICH-FOS and compared scores with regard to mortality and poor functional outcome at 3-month after ICH.

\& $\mathrm{P}$ value of comparing paired AUROC with Delong's method.

Abbreviation: ICH, Intracerebral Hemorrhage; AUROC, Area Under the Receiver Operating Characteristic Curve; $\mathrm{Cl}$, Confidence Interval; PPV, Positive Predictive Value; NPV, Negative Predictive Value. 


\begin{tabular}{|c|c|c|c|c|c|c|c|c|c|c|}
\hline & AUROC & $95 \% \mathrm{Cl}$ & $\stackrel{\Delta}{A U R O C^{*}}$ & $\begin{array}{l}\text { P } \\
\text { value }^{\&}\end{array}$ & $\begin{array}{l}\text { Youden } \\
\text { Index }\end{array}$ & Cutoff & Sensitivity & Specificity & PPV & NPV \\
\hline $\begin{array}{l}\text { The } \\
\text { simplified } \\
\text { ICH score } \\
(2009)\end{array}$ & 0.786 & $\begin{array}{l}0.765- \\
0.806\end{array}$ & 0.094 & $\begin{array}{l}< \\
0.001\end{array}$ & 0.469 & 6 & 0.675 & 0.794 & 0.802 & 0.665 \\
\hline $\begin{array}{l}\text { The EDICH } \\
\text { score } \\
(2014)\end{array}$ & 0.772 & $\begin{array}{l}0.751- \\
0.793\end{array}$ & 0.108 & $\begin{array}{l}< \\
0.001\end{array}$ & 0.446 & 1 & 0.659 & 0.786 & 0.793 & 0.652 \\
\hline $\begin{array}{l}\text { Broderick's } \\
\text { score } \\
(1993)\end{array}$ & 0.771 & $\begin{array}{l}0.749- \\
0.791\end{array}$ & 0.109 & $\begin{array}{l}< \\
0.001\end{array}$ & 0.453 & $\ldots$ & 0.713 & 0.740 & 0.772 & 0.677 \\
\hline $\begin{array}{l}\text { The } \\
\text { original } \\
\text { ICH Score } \\
(2001)\end{array}$ & 0.768 & $\begin{array}{l}0.746- \\
0.789\end{array}$ & 0.112 & $\begin{array}{l}< \\
0.001\end{array}$ & 0.429 & 1 & 0.586 & 0.842 & 0.821 & 0.623 \\
\hline $\begin{array}{l}\text { The ICH- } \\
\text { GS (2007) }\end{array}$ & 0.768 & $\begin{array}{l}0.746- \\
0.789\end{array}$ & 0.112 & $\dot{0} 001$ & 0.429 & 1 & 0.586 & 0.842 & 0.821 & 0.623 \\
\hline $\begin{array}{l}\text { Huang's } \\
\text { score } \\
(2008)\end{array}$ & 0.766 & $\begin{array}{l}0.744- \\
0.787\end{array}$ & 0.114 & $\begin{array}{l}< \\
0.001\end{array}$ & 0.456 & 1 & 0.627 & 0.829 & 0.820 & 0.643 \\
\hline $\begin{array}{l}\text { Tuhrim's } \\
\text { Eq. (1991) }\end{array}$ & 0.741 & $\begin{array}{l}0.719- \\
0.763\end{array}$ & 0.139 & $\dot{0} 001$ & 0.379 & $\ldots$ & 0.478 & 0.901 & 0.856 & 0.583 \\
\hline $\begin{array}{l}\text { Ho's score } \\
\text { (2016) }\end{array}$ & 0.739 & $\begin{array}{l}0.716- \\
0.760\end{array}$ & 0.141 & $<.001$ & 0.432 & $\ldots$ & 0.611 & 0.821 & 0.808 & 0.631 \\
\hline $\begin{array}{l}\text { The } \\
\text { Kinshasa } \\
\text { ICH score } \\
\text { (2012) }\end{array}$ & 0.721 & $\begin{array}{l}0.698- \\
0.743\end{array}$ & 0.159 & $\begin{array}{l}<.001 \\
0.00\end{array}$ & 0.391 & 6 & 0.570 & 0.821 & 0.797 & 0.608 \\
\hline $\begin{array}{l}\text { Shaya's } \\
\text { score } \\
(2005)\end{array}$ & 0.716 & $\begin{array}{l}0.693- \\
0.739\end{array}$ & 0.164 & $\begin{array}{l}< \\
0.001\end{array}$ & 0.379 & 2 & 0.575 & 0.804 & 0.784 & 0.605 \\
\hline $\begin{array}{l}\text { Weimar' } \\
\text { equation-I } \\
\text { (2006) }\end{array}$ & 0.691 & $\begin{array}{l}0.667- \\
0.714\end{array}$ & 0.189 & $\begin{array}{l}< \\
0.001\end{array}$ & 0.271 & $\ldots$ & 0.655 & 0.615 & 0.678 & 0.592 \\
\hline $\begin{array}{l}\text { Safatli's } \\
\text { score } \\
(2016)\end{array}$ & 0.602 & $\begin{array}{l}0.577- \\
0.626\end{array}$ & 0.278 & $\begin{array}{l}< \\
0.001\end{array}$ & 0.203 & 0 & 0.227 & 0.975 & 0.921 & 0.506 \\
\hline \multicolumn{11}{|c|}{$\begin{array}{l}{ }^{\star} \triangle \mathrm{AUROC} \text { denotes the difference in AUROC between the ICH-FOS and compared scores with regard to mortality and poor } \\
\text { functional outcome at 3-month after ICH. }\end{array}$} \\
\hline \multicolumn{11}{|c|}{ \& $\mathrm{P}$ value of comparing paired AUROC with Delong's method. } \\
\hline $\begin{array}{l}\text { bbreviatic } \\
\text { onfidence }\end{array}$ & $\mathrm{H}$, Intr & bral 1 & rrhage & C, A & nder $\mathrm{t}$ & ceiv & erating $\mathrm{Ch}$ & cteristic C & $\mathrm{Cl}$ & \\
\hline
\end{tabular}


Table 3

Calibration of ICH risk models with regard to mortality and functional outcome at 3-month after ICH $(n=1575)$

\begin{tabular}{|c|c|c|c|c|c|c|c|}
\hline \multirow[t]{2}{*}{$\begin{array}{l}\text { International ICH } \\
\text { models }\end{array}$} & \multicolumn{3}{|c|}{$\begin{array}{l}\text { Goodness of fit test with regard } \\
\text { to } \\
\text { mortality at 3-month after onset }\end{array}$} & \multirow[t]{2}{*}{$\begin{array}{l}\text { International ICH } \\
\text { models }\end{array}$} & \multicolumn{3}{|c|}{$\begin{array}{l}\text { Goodness of fit test with regard to } \\
\text { poor functional outcome (mRS } \geq 3 \text { ) } \\
\text { at 3-month after onset }\end{array}$} \\
\hline & $\begin{array}{l}P \\
\text { value }\end{array}$ & $\begin{array}{l}\text { Cox and } \\
\text { Snell } \mathrm{R}^{2}\end{array}$ & $\begin{array}{l}\text { Nagelkerke } \\
\mathrm{R}^{2}\end{array}$ & & $\begin{array}{l}\mathrm{P} \\
\text { value }\end{array}$ & $\begin{array}{l}\text { Cox and } \\
\text { Snell } \mathbf{R}^{2}\end{array}$ & $\begin{array}{l}\text { Nagelkerke } \\
\mathrm{R}^{2}\end{array}$ \\
\hline The ICH-FOS (2013) & $\overrightarrow{0.05}$ & 0.249 & 0.376 & $\begin{array}{l}\text { The ICH-FOS } \\
(2013)\end{array}$ & $\overrightarrow{0.05}$ & 0.399 & 0.535 \\
\hline $\begin{array}{l}\text { The modified ICH } \\
\text { Score (2003) }\end{array}$ & $\overrightarrow{0.05}$ & 0.237 & 0.367 & max-ICH (2017) & $\overrightarrow{0.05}$ & 0.373 & 0.50 \\
\hline $\begin{array}{l}\text { The GP on STAGE } \\
\text { score }(2006)\end{array}$ & $\begin{array}{l}> \\
0.05\end{array}$ & 0.232 & 0.365 & $\begin{array}{l}\text { Hallevy's score } \\
(2002)\end{array}$ & $\overrightarrow{0.05}$ & 0.337 & 0.45 \\
\hline $\begin{array}{l}\text { CAA- ICH score } \\
(2016)\end{array}$ & $\begin{array}{l}> \\
0.05\end{array}$ & 0.229 & 0.354 & $\begin{array}{l}\text { The modified ICH } \\
\text { Score (2003) }\end{array}$ & $\overrightarrow{0.05}$ & 0.301 & 0.403 \\
\hline Lisk's Eq. (1994) & $\begin{array}{l}> \\
0.05\end{array}$ & 0.224 & 0.347 & $\begin{array}{l}\text { The IVH score } \\
(2010)\end{array}$ & $\overrightarrow{0.05}$ & 0.287 & 0.384 \\
\hline $\begin{array}{l}\text { The ICH index } \\
\text { (2012) }\end{array}$ & $\begin{array}{l}> \\
0.05\end{array}$ & 0.217 & 0.336 & $\begin{array}{l}\text { The FUNC Score } \\
(2008)\end{array}$ & $\overrightarrow{0.05}$ & 0.273 & 0.366 \\
\hline $\begin{array}{l}\text { The original ICH } \\
\text { Score (2001) }\end{array}$ & $\overrightarrow{0.05}$ & 0.193 & 0.299 & $\begin{array}{l}\text { CAA- ICH score } \\
(2016)\end{array}$ & $\overrightarrow{0.05}$ & 0.264 & 0.354 \\
\hline The ICH-GS (2007) & $\begin{array}{l}> \\
0.05\end{array}$ & 0.193 & 0.299 & $\begin{array}{l}\text { The MICH Score } \\
(2008)\end{array}$ & $\overrightarrow{0.05}$ & 0.263 & 0.351 \\
\hline $\begin{array}{l}\text { Weimar' equation-I } \\
\text { (2006) }\end{array}$ & $\overrightarrow{0.05}$ & 0.044 & 0.068 & $\begin{array}{l}\text { The ICH index } \\
(2012)\end{array}$ & $\overrightarrow{0.05}$ & 0.234 & 0.313 \\
\hline $\begin{array}{l}\text { The Essen ICH score } \\
(2006)\end{array}$ & $\begin{array}{l}< \\
0.05\end{array}$ & 0.245 & 0.379 & $\begin{array}{l}\text { Weimar' equation-I } \\
(2006)\end{array}$ & $\overrightarrow{0.05}$ & 0.109 & 0.146 \\
\hline $\begin{array}{l}\text { The simplified ICH } \\
\text { score (2009) }\end{array}$ & $\begin{array}{l}< \\
0.05\end{array}$ & 0.243 & 0.375 & $\begin{array}{l}\text { The Essen ICH } \\
\text { score }(2006)\end{array}$ & $\begin{array}{l}<.05 \\
0.05\end{array}$ & 0.363 & 0.486 \\
\hline $\begin{array}{l}\text { The EDICH score } \\
\text { (2014) }\end{array}$ & $\begin{array}{l}< \\
0.05\end{array}$ & 0.242 & 0.374 & ICHOP (2018) & $<.05$ & 0.346 & 0.463 \\
\hline $\begin{array}{l}\text { Weimar' equation-II } \\
(2009)\end{array}$ & $\begin{array}{l}<.05 \\
0.05\end{array}$ & 0.241 & 0.373 & $\begin{array}{l}\text { Weimar' equation-I } \\
\text { (2006) }\end{array}$ & $<.05$ & 0.306 & 0.410 \\
\hline $\begin{array}{l}\text { Huang's score } \\
\text { (2008) }\end{array}$ & $\dot{0} 0.05$ & 0.231 & 0.358 & $\begin{array}{l}\text { The GP on STAGE } \\
\text { score }(2006)\end{array}$ & $\dot{0} 0.05$ & 0.291 & 0.39 \\
\hline ICHOP (2018) & $\begin{array}{l}< \\
0.05\end{array}$ & 0.227 & 0.351 & Lisk's Eq. (1994) & $<.05$ & 0.284 & 0.38 \\
\hline $\begin{array}{l}\text { Broderick's score } \\
(1993)\end{array}$ & $\dot{0} 0.05$ & 0.215 & 0.333 & Mase's Eq. (1995) & $\dot{0.05}$ & 0.271 & 0.363 \\
\hline $\begin{array}{l}\text { The FUNC Score } \\
(2008)\end{array}$ & $\begin{array}{l}< \\
0.05\end{array}$ & 0.209 & 0.323 & $\begin{array}{l}\text { The simplified ICH } \\
\text { score (2009) }\end{array}$ & $\begin{array}{l}<.05 \\
0.05\end{array}$ & 0.26 & 0.348 \\
\hline Mase's Eq. (1995) & $\begin{array}{l}<.05 \\
0.05\end{array}$ & 0.193 & 0.299 & $\begin{array}{l}\text { Huang's score } \\
\text { (2008) }\end{array}$ & $<.05$ & 0.249 & 0.333 \\
\hline max-ICH (2017) & $\begin{array}{l}< \\
0.05\end{array}$ & 0.193 & 0.298 & $\begin{array}{l}\text { The EDICH score } \\
(2014)\end{array}$ & $\begin{array}{l}< \\
0.05\end{array}$ & 0.239 & 0.321 \\
\hline
\end{tabular}

Abbreviation: ICH, Intracerebral Hemorrhage; mRS, modified Rankin Scale. 


\begin{tabular}{|c|c|c|c|c|c|c|c|}
\hline \multirow[t]{2}{*}{$\begin{array}{l}\text { International ICH } \\
\text { models }\end{array}$} & \multicolumn{3}{|c|}{$\begin{array}{l}\text { Goodness of fit test with regard } \\
\text { to } \\
\text { mortality at 3-month after onset }\end{array}$} & \multirow[t]{2}{*}{$\begin{array}{l}\text { International ICH } \\
\text { models }\end{array}$} & \multicolumn{3}{|c|}{$\begin{array}{l}\text { Goodness of fit test with regard to } \\
\text { poor functional outcome (mRS } \geq 3 \text { ) } \\
\text { at 3-month after onset }\end{array}$} \\
\hline & $\begin{array}{l}P \\
\text { value }\end{array}$ & $\begin{array}{l}\text { Cox and } \\
\text { Snell } \mathrm{R}^{2}\end{array}$ & $\begin{array}{l}\text { Nagelkerke } \\
\mathrm{R}^{2}\end{array}$ & & $\begin{array}{l}P \\
\text { value }\end{array}$ & $\begin{array}{l}\text { Cox and } \\
\text { Snell R }\end{array}$ & $\begin{array}{l}\text { Nagelkerke } \\
\mathrm{R}^{2}\end{array}$ \\
\hline $\begin{array}{l}\text { The IVH score } \\
(2010)\end{array}$ & $\hat{0}_{0.05}$ & 0.189 & 0.292 & $\begin{array}{l}\text { The original ICH } \\
\text { Score }(2001)\end{array}$ & $<.05$ & 0.233 & 0.312 \\
\hline $\begin{array}{l}\text { The MICH Score } \\
\text { (2008) }\end{array}$ & $\dot{0} 0.05$ & 0.185 & 0.286 & The ICH-GS (2007) & $<.05$ & 0.233 & 0.312 \\
\hline Ho's score (2016) & $\begin{array}{l}< \\
0.05\end{array}$ & 0.170 & 0.263 & $\begin{array}{l}\text { Broderick's score } \\
(1993)\end{array}$ & $\dot{0} 05$ & 0.217 & 0.291 \\
\hline $\begin{array}{l}\text { Hallevy's score } \\
(2002)\end{array}$ & $\dot{0.05}$ & 0.147 & 0.228 & Ho's score (2016) & $<.05$ & 0.215 & 0.288 \\
\hline Tuhrim's Eq. (1991) & $\begin{array}{l}<.05 \\
0.05\end{array}$ & 0.143 & 0.221 & Tuhrim's Eq. (1991) & $\dot{0.05}$ & 0.201 & 0.268 \\
\hline $\begin{array}{l}\text { The Kinshasa ICH } \\
\text { score (2012) }\end{array}$ & $\hat{0}_{0.05}$ & 0.077 & 0.119 & $\begin{array}{l}\text { The Kinshasa ICH } \\
\text { score (2012) }\end{array}$ & $<.05$ & 0.161 & 0.216 \\
\hline $\begin{array}{l}\text { Shaya's score } \\
(2005)\end{array}$ & $\begin{array}{l}<.05 \\
0.05\end{array}$ & 0.059 & 0.091 & $\begin{array}{l}\text { Shaya's score } \\
(2005)\end{array}$ & $<.05$ & 0.16 & 0.214 \\
\hline $\begin{array}{l}\text { Safatli's score } \\
\text { (2016) }\end{array}$ & $\begin{array}{l}< \\
0.05\end{array}$ & 0.051 & 0.079 & $\begin{array}{l}\text { Safatli's score } \\
(2016)\end{array}$ & $\begin{array}{l}< \\
0.05\end{array}$ & 0.098 & 0.131 \\
\hline
\end{tabular}

Abbreviation: ICH, Intracerebral Hemorrhage; mRS, modified Rankin Scale.

Calibration. The predicted and observed risk according to 10 deciles of the predicted risk of mortality at 3-month after ICH was plotted (An additional figure shows this in more detail [see Additional Fig. 4]). The results of Hosmer-Lemeshow test are shown in Table 3. Nine models have a significance level of Hosmer-Lemeshow test greater than 0.05, as indicated that the observed values are not statistically different from the expected values. Among them, the risk models with top three largest Cox and Snell R-square were the ICH-FOS, modified ICH Score and GP on STAGE score, respectively. Similar results were verified for mortality at 1-month and 1-year after ICH (Additional tables shows this in more detail [see Additional table4-5]).

\section{Predictive Performance For Poor Functional Outcome After Ich}

Discrimination. For predicting poor functional outcome (mRS $\geq 3$ ) at 3-month after ICH, AUROC of 27 ICH risk models ranged from 0.602 to 0.880 . The ICH-FOS score (AUROC $=0.880 ; 95 \% \mathrm{Cl}=0.864-0.897 ; \mathrm{P}<0.001)$, Essen ICH score $(\mathrm{AUROC}=0.862$; $95 \% \mathrm{Cl}=0.843-0.878 ; \mathrm{P}<0.001)$, max-ICH score (AUROC $=0.862 ; 95 \% \mathrm{Cl}=0.844-0.878 ; \mathrm{P}<0.001$ ), Weimer's equation-II $(A U R O C=0.853 ; 95 \% \mathrm{Cl}=0.835-0.872 ; \mathrm{P}<0.001)$ and ICHOP scores $(\mathrm{AUROC}=0.850 ; 95 \% \mathrm{Cl}=0.832-0.867 ; \mathrm{P}<0.001)$ demonstrated excellent discrimination with AUROC between 0.85 and 0.89 . Six risk models showed good discrimination with AUROC between 0.80 and 0.84 , including the Hallevy's score, modified ICH Score, Lisk's equation, GP on STAGE score, Mase's equation and IVH score. Ten risk models demonstrated moderate discrimination with AUROC between 0.75 and 0.79 (Table 2) (An additional figure shows this in more detail [see Additional Fig. 3]). The sensitivity, specificity, PPV, NPV and maximum Youden Index of ICH risk models with regard to poor functional outcome at 3-month after onset are shown in Table 2. The $\mathrm{ICH}-\mathrm{FOS}$ showed the maximum Youden Index. In pairwise comparison with other prediction models, the ICH-FOS was superior in predicting poor functional outcome at 3-month after ICH (all P<0.001). Similar results were found with regard to poor functional outcome at 1-month and 1-year after ICH (Additional tables show this in more detail [see Additional Table2-3]). 
Calibration. The predicted and observed risk according to 10 deciles of the predicted risk of poor functional outcome at 3month after ICH was plotted in supplementary Fig. 5 (An additional figure shows this in more detail [see Additional Fig. 5]). The results of Hosmer-Lemeshow test are shown in Table 3. Ten risk models have a significance level of Hosmer-Lemeshow test greater than 0.05. Among them, the models with top three largest Cox and Snell R-square of Hosmer-Lemeshow test were the ICH-FOS, max-ICH and Hallevy's score, respectively (Table 3). Similar results were found for poor functional outcome at 1month and 1-year after ICH (Additional tables show this in more detail [see Additional table 5-7]).

\section{Sensitivity Analysis}

We completed prespecified subgroup analyses by age ( $\leq 59$ vs. $\geq 60$ ), gender (female vs. male), hematoma location (superatentorial vs. infratentorial), hematoma volume ( $\leq 29 \mathrm{vs.} \geq 30 \mathrm{ml}$ ), medical care withdrawal (yes vs. no) and surgical treatment (yes vs. no). Although several risk models demonstrated good or excellent discrimination in these subgroups, the ICH-FOS steadily showed the highest AUROC for poor functional outcome (mRS $\geq 3$ ) at 3-month (Fig. 2A) and 1-year (Fig. 2B) after onset.

\section{Discussion}

In the study, we systematically compared discrimination and calibration of $27 \mathrm{ICH}$ risk models with regard to mortality and poor functional outcome at 1-month, 3-month and 1-year after $\mathrm{ICH}$, respectively. For predicting 3-month mortality after ICH, AUROC of $27 \mathrm{ICH}$ risk models ranged from 0.604 to 0.856 . Among them, one model showed excellent discrimination and fifteen models demonstrated good discrimination with regard to mortality at 3-month after ICH. For predicting poor functional outcome (mRS $\geq 3)$ at 3-month after ICH, AUROC of $27 \mathrm{ICH}$ risk models ranged from 0.602 to 0.880 . Five model showed excellent discrimination and six models demonstrated good discrimination for poor functional outcome at 3-month after ICH. In pairwise comparison, the ICH-FOS showed statistically better discrimination than other ICH models for both mortality and poor functional outcomes at 3-month after ICH. Similar results were verified for mortality and poor functional outcome at 1month and 1-year after $\mathrm{ICH}$.

A lot of literature conveyed that do-not-resuscitate order and withdraw of care contribute to worse outcomes in patients with $\mathrm{ICH}$ and majority of available ICH risk models are hindered by this self-fulfilling prophecy[38, 39]. The ideal way to precisely define prognosis after ICH would be to assess it in a cohort in which patients received full support, irrespective of the perceived probable outcome. The Beijing Registration of Intracerebral Hemorrhage had an overall low rate of withdrawal of care $(6.6 \%)$ and allowed us to get closer to overcoming the self-fulfilling prophecy prejudice.

For a clinical risk model to become effective and widely used, it must be accurate and reliable in risk-stratification and outcome prediction. Our study indicated that among $27 \mathrm{ICH}$ risk models, seventeen models had good or excellent discrimination (AUROC $\geq 0.80$ ) for predicting mortality at 3-month after ICH; meanwhile, eleven models showed good or excellent discrimination for poor functional outcome ( $m R S \geq 3$ ) at 3-month after ICH (Table 2). Similar results were found for predicting mortality and poor functional outcome at 1-month and 1-year after ICH (Supplementary table 2-3). These results indicated that several ICH risk models are effective for predicting mortality and functional outcomes after ICH, which would be useful for data-driven discussion with patients or families, personalized care and clinical trial.

With several ICH prognostic models available, it is becoming increasingly difficult for clinician and researcher to determine which risk models provide optimal predictability and reliability in clinical practice and clinical trial. In pairwise comparison, the $\mathrm{ICH}-F O S$ showed statistically better discrimination than other ICH risk models for both mortality (all $\mathrm{P}<0.05)$ and poor functional outcome (all $\mathrm{P}<0.001$ ) at 3-month after $\mathrm{ICH}$. Similar results were verified for predicting mortality and poor functional outcome at 1-month and 1-year after ICH (Supplementary table 2-3). In addition, in subgroup analysis, the ICHFOS steadily showed the highest AUROC in prespecified subgroups for poor functional outcome at 3-month and 1-year after ICH (Fig. 2). In calibration analysis, the ICH-FOS showed the largest Cox and Snell R-square of the Hosmer-Lemeshow goodness of fit test for both mortality and poor functional outcome at 1-month, 3-month and 1-year after ICH. These results

Page 14/22 
were consistent with the original study for developing the ICH-FOS based on the China National Stroke Registry (CNSR)[30]. Together, these results indicated that the ICH-FOS had significantly better discrimination and calibration than compared models for both mortality and poor functional outcome after ICH. Though it is promising, caution need to be taken when interpreting the results: first, the study populations for derivation and validation of these ICH models are different. The baseline characteristics of our study were different from those of western cohorts, such as with younger age of ICH onset, less severity of neurological deficit, smaller hematoma volume, fewer intraventricular extension and lower rate of withdraw of care. Second, the intended outcome (functional outcome vs. mortality), timing (1-month, 3-month, 6-month and 1-year after $\mathrm{ICH}$ ) and assessment tools (GOS vs. BI vs. mRS) are different for existing ICH models. Finally, there might be complex genetic, social, economic factors as well as regional management philosophies and preferences that are difficult to account for when risk models are developed or applied to a distinct population. These ICH models need to be further validated in more populations and larger samples in the future.

Despite advances in medical knowledge, treatment for ICH remains strictly supportive with not many evidence-based interventions currently available. Medical and surgical treatment, such as blood pressure control[40, 41], hematoma evacuation[42-44], hemostatic therapy[45], neuroprotection[46] have not shown definite benefit in improving ICH functional outcomes. It is the time that we need to rethink about the way how we conduct a clinical trial for improving ICH functional outcome. Currently, we have come into a new era of precision medicine. According to the Precision Medicine Initiative, precision medicine is a medical model that proposes the customization of healthcare, with medical treatment or prevention being tailored to individual patient[47]. In precision medicine, patients are stratified by potential risk and treatment or prevention strategies are developed for patients with specific risk stratification. On the country, in traditional medicine model, patients were grouped by symptoms and signs and treatment or prevention strategies are developed for the average person (one size fit all), with less consideration for the differences between individuals. Prior ICH clinical trials selected patients mainly based on hematoma volume, hematoma location and time window of symptom onset[40-46]. In this way, it is inevitable to include patients with unbalanced, too high, or too low risk of developing poor functional outcome in these clinical trials (An additional figure shows this in more detail [see Additional Fig. 6A]). Inspired by precision medicine model, we could use validated prognostic models to stratify patients by potential risk of developing poor functional outcome after ICH and then test specific intervention in different risk stratifications (An additional figure shows this in more detail [see Additional Fig. 6B]). ICH trials conducted in this way will allow researchers to clarify more accurately which treatment or prevention strategies will work in which risk stratification patients.

Our study has strength and limitation. To the best of our knowledge, we are the first to systematically compare predictive performance of $27 \mathrm{ICH}$ risk models with regard to mortality and functional outcome after ICH in a large independent cohort. The finding of the study will provide important information on selecting prognostic tools for personalized care and clinical trial in $\mathrm{ICH}$. However, our study also has limitation that deserve comment: First, central follow-up blinded to baseline variables was made by telephone interview by trained interviewers based on a standardized interview protocol. For patients who lost to follow-up, we made telephone follow-up interview once a week for three times. Although a lot of effort has been done, there is still a relatively high rate of patients with missing follow-up information during 1-year after ICH. We compared the baseline characteristics of patients included and those excluded and they were not statistically different in admission NIHSS score, GCS score, hematoma volume and withdraw of care. Second, we cannot have all elements required for all ICH models and several ICH risk models cannot be externally validated in the study. Meanwhile, some risk models developed based on machine learning were not included in the study as well. Third, our study included only hospitalized patients and those patients died in emergency room or treated in outpatient clinics were not included. Meanwhile, like most registries, our registry required informed consent and selection bias was inevitable. Finally, validation cohorts originated from Asian population and the ICH models needed to be further validated in different populations.

\section{Conclusion}

Several risk models are externally validated to be effective for risk stratification and outcome prediction after ICH, especially the ICH-FOS, which would be useful tools for personalized care and clinical trials in ICH.

Page $15 / 22$ 


\section{List Of Abbreviations}

Intracerebral hemorrhage (ICH)

Transparent reporting of a multivariable prediction model for individual prognosis or diagnosis (TRIPOD) guideline

modified Rankin Scale (mRS)

Institutional Review Board (IRB)

electronic case report form (eCRF)

National Institutes of Health Stroke Scale score (NIHSS)

Glasgow Coma Scale (GCS)

Length of hospital stay (LOS)

Standard deviation (SD)

Interquartile range (IQR)

Area under the receiver operating characteristic curve (AUROC)

Positive predict value (PPV)

Negative predictive value (NPV)

China National Stroke Registry (CNSR)

The ICH Functional Outcome Score (ICH-FOS)

\section{Declarations}

The study protocol was approved by the Institutional Review Board (IRB) of the Beijing Tiantan Hospital (KY2014-023-02).

\section{All authors are consent for publication.}

Availability of data and materials: the corresponding author has the responsibility on behalf of Beijing Registration of Intracerebral Hemorrhage investigators and has full access to the final database. The datasets used and/or analysed during the current study are available from the corresponding author on reasonable request

\section{Competing interests}

Dr. Ruijun Ji reports no disclosures.

Dr. Wenjuan Wang reports no disclosures.

Dr. Xinyu Liu reports no disclosures.

Dr. Linlin Wang reports no disclosures.

Dr. Ruixuan Jiang reports no disclosures.

Dr. Runhua Zhang reports no disclosures. 
Dr. Dandan Wang reports no disclosures.

Dr. Jiaokun Jia reports no disclosures.

Dr. Hao Feng reports no disclosures.

Dr. Zeyu Ding reports no disclosures.

Dr. Yanfang Liu reports no disclosures.

Dr. Gaifen Liu reports no disclosures.

Dr. Jingjing Lu reports no disclosures.

Dr. Yi Ju reports no disclosures.

Dr. Xingquan Zhao reports no disclosures.

\section{Funding}

This study was sponsored by Capital health research and development of special (2011-2004-03) and Beijing Municipal Science \& Technology Commission (Z131107002213009). This study was partially supported by the Nova Program of Beijing Science and Technology Commission (2008B30), National Natural Science Foundation of China $(81471208,81641162)$, Beijing high-level healthy human resource project (014-3-033) and Shandong Province Key Innovation Project (2019JZZY020901).

\section{Authors' contributions}

Conception and design: Ruijun Ji, Xingquan Zhao.

Acquisition of data: Beijing Registration of Intracerebral Hemorrhage investigators.

Analysis or interpretation of data: All authors.

Drafting the manuscript: Ruijun Ji, Xinyu Liu, Linlin Wang.

Revising the manuscript for important intellectual content: Ruijun Ji, Xingquan Zhao, Gaifen Liu, Yi Ju.

Statistical analysis: Runhua Zhang, Gaifen Liu, Ruijun Ji.

Obtaining funding: Xingquan Zhao, Ruijun Ji.

Supervision: Xingquan Zhao.

\section{References}

1. Virani SS, Alonso A, Benjamin EJ, Bittencourt MS, Callaway CW, Carson AP, Chamberlain AM, Chang AR, Cheng S, Delling FN et al: Heart Disease and Stroke Statistics-2020 Update: A Report From the American Heart Association. Circulation 2020, 141(9):e139-e596.

2. Wang W, Jiang B, Sun H, Ru X, Sun D, Wang L, Wang L, Jiang Y, Li Y, Wang Y et al: Prevalence, Incidence, and Mortality of Stroke in China: Results from a Nationwide Population-Based Survey of 480687 Adults. Circulation 2017, 135(8):759771.

3. Hemphill JC, 3rd, Greenberg SM, Anderson CS, Becker K, Bendok BR, Cushman M, Fung GL, Goldstein JN, Macdonald RL, Mitchell PH et al: Guidelines for the Management of Spontaneous Intracerebral Hemorrhage: A Guideline for Healthcare

Page $17 / 22$ 
Professionals From the American Heart Association/American Stroke Association. Stroke 2015, 46(7):2032-2060.

4. Steiner T, Al-Shahi Salman R, Beer R, Christensen H, Cordonnier C, Csiba L, Forsting M, Harnof S, Klijn CJ, Krieger D et al: European Stroke Organisation (ESO) guidelines for the management of spontaneous intracerebral hemorrhage. Int $\mathrm{J}$ Stroke 2014, 9(7):840-855.

5. Gregorio T, Pipa S, Cavaleiro P, Atanasio G, Albuquerque I, Chaves PC, Azevedo L: Prognostic models for intracerebral hemorrhage: systematic review and meta-analysis. BMC Med Res Methodo/ 2018, 18(1):145.

6. Hemphill JC, 3rd, Bonovich DC, Besmertis L, Manley GT, Johnston SC: The ICH score: a simple, reliable grading scale for intracerebral hemorrhage. Stroke 2001, 32(4):891-897.

7. Moons KG, Altman DG, Reitsma JB, loannidis JP, Macaskill P, Steyerberg EW, Vickers AJ, Ransohoff DF, Collins GS: Transparent Reporting of a multivariable prediction model for Individual Prognosis or Diagnosis (TRIPOD): explanation and elaboration. Ann Intern Med 2015, 162(1):W1-73.

8. Collins GS, Reitsma JB, Altman DG, Moons KG: Transparent reporting of a multivariable prediction model for individual prognosis or diagnosis (TRIPOD): the TRIPOD statement. BMJ 2015, 350:g7594.

9. Sacco RL, Kasner SE, Broderick JP, Caplan LR, Connors JJ, Culebras A, Elkind MS, George MG, Hamdan AD, Higashida RT et al: An updated definition of stroke for the 21st century: a statement for healthcare professionals from the American Heart Association/American Stroke Association. Stroke 2013, 44(7):2064-2089.

10. Kothari RU, Brott T, Broderick JP, Barsan WG, Sauerbeck LR, Zuccarello M, Khoury J: The ABCs of measuring intracerebral hemorrhage volumes. Stroke 1996, 27(8):1304-1305.

11. Tuhrim S, Dambrosia JM, Price TR, Mohr JP, Wolf PA, Hier DB, Kase CS: Intracerebral hemorrhage: external validation and extension of a model for prediction of 30-day survival. Ann Neurol 1991, 29(6):658-663.

12. Broderick JP, Brott TG, Duldner JE, Tomsick T, Huster G: Volume of intracerebral hemorrhage. A powerful and easy-to-use predictor of 30-day mortality. Stroke 1993, 24(7):987-993.

13. Lisk DR, Pasteur W, Rhoades H, Putnam RD, Grotta JC: Early presentation of hemispheric intracerebral hemorrhage: prediction of outcome and guidelines for treatment allocation. Neurology 1994, 44(1):133-139.

14. Mase G, Zorzon M, Biasutti E, Tasca G, Vitrani B, Cazzato G: Immediate prognosis of primary intracerebral hemorrhage using an easy model for the prediction of survival. Acta Neurol Scand 1995, 91(4):306-309.

15. Hallevy C, Ifergane G, Kordysh E, Herishanu Y: Spontaneous supratentorial intracerebral hemorrhage. Criteria for shortterm functional outcome prediction. J Neuro/ 2002, 249(12):1704-1709.

16. Cheung RT, Zou LY: Use of the original, modified, or new intracerebral hemorrhage score to predict mortality and morbidity after intracerebral hemorrhage. Stroke 2003, 34(7):1717-1722.

17. Shaya M, Dubey A, Berk C, Gonzalez-Toledo E, Zhang J, Caldito G, Nanda A: Factors influencing outcome in intracerebral hematoma: a simple, reliable, and accurate method to grade intracerebral hemorrhage. Surg Neurol 2005, 63(4):343-348; discussion 348.

18. Weimar C, Benemann J, Diener HC, German Stroke Study C: Development and validation of the Essen Intracerebral Haemorrhage Score. J Neurol Neurosurg Psychiatry 2006, 77(5):601-605.

19. Weimar C, Roth M, Willig V, Kostopoulos P, Benemann J, Diener HC: Development and validation of a prognostic model to predict recovery following intracerebral hemorrhage. J Neurol 2006, 253(6):788-793.

20. Poungvarin N, Suwanwela NC, Venketasubramanian N, Wong LK, Navarro JC, Bitanga E, Yoon BW, Chang HM, Alam SM, Asian Stroke Advisory P: Grave prognosis on spontaneous intracerebral haemorrhage: GP on STAGE score. J Med Assoc Thai 2006, 89 Suppl 5:S84-93.

21. Ruiz-Sandoval JL, Chiquete E, Romero-Vargas S, Padilla-Martinez JJ, Gonzalez-Cornejo S: Grading scale for prediction of outcome in primary intracerebral hemorrhages. Stroke 2007, 38(5):1641-1644.

22. Rost NS, Smith EE, Chang Y, Snider RW, Chanderraj R, Schwab K, FitzMaurice E, Wendell L, Goldstein JN, Greenberg SM et al: Prediction of functional outcome in patients with primary intracerebral hemorrhage: the FUNC score. Stroke 2008, 
39(8):2304-2309.

23. Cho DY, Chen CC, Lee WY, Lee HC, Ho LH: A new Modified Intracerebral Hemorrhage score for treatment decisions in basal ganglia hemorrhage-a randomized trial. Crit Care Med 2008, 36(7):2151-2156.

24. Huang BR, Liao CC, Huang WH, Hsu YH, Hsu JC, Yen HC, Lin CL: Prognostic factors of spontaneous intracerebral haemorrhage in haemodialysis patients and predictors of 30-day mortality. Intern Med J 2008, 38(7):568-574.

25. Chuang YC, Chen YM, Peng SK, Peng SY: Risk stratification for predicting 30-day mortality of intracerebral hemorrhage. Int J Qual Health Care 2009, 21(6):441-447.

26. Weimar C, Ziegler A, Sacco RL, Diener HC, Konig IR, investigators V: Predicting recovery after intracerebral hemorrhagean external validation in patients from controlled clinical trials. J Neuro/ 2009, 256(3):464-469.

27. Stein M, Luecke M, Preuss M, Boeker DK, Joedicke A, Oertel MF: Spontaneous intracerebral hemorrhage with ventricular extension and the grading of obstructive hydrocephalus: the prediction of outcome of a special life-threatening entity. Neurosurgery 2010, 67(5):1243-1251; discussion 1252.

28. Tshikwela ML, Longo-Mbenza B: Spontaneous intracerebral hemorrhage: Clinical and computed tomography findings in predicting in-hospital mortality in Central Africans. J Neurosci Rural Pract 2012, 3(2):115-120.

29. Li YF, Luo J, Li Q, Jing YJ, Wang RY, Li RS: A new simple model for prediction of hospital mortality in patients with intracerebral hemorrhage. CNS Neurosci Ther 2012, 18(6):482-486.

30. Ji R, Shen H, Pan Y, Wang P, Liu G, Wang Y, Li H, Zhao X, Wang Y, China National Stroke Registry i: A novel risk score to predict 1-year functional outcome after intracerebral hemorrhage and comparison with existing scores. Crit Care 2013, 17(6):R275.

31. Zis P, Leivadeas P, Michas D, Kravaritis D, Angelidakis P, Tavernarakis A: Predicting 30-day case fatality of primary inoperable intracerebral hemorrhage based on findings at the emergency department. J Stroke Cerebrovasc Dis 2014, 23(7):1928-1933.

32. Lei C, Wu B, Liu M, Zhang S, Yuan R: Cerebral Amyloid Angiopathy-related Intracerebral Hemorrhage Score For Predicting Outcome. Curr Neurovasc Res 2016, 13(2):156-162.

33. Ho WM, Lin JR, Wang HH, Liou CW, Chang KC, Lee JD, Peng TY, Yang JT, Chang YJ, Chang CH et al: Prediction of inhospital stroke mortality in critical care unit. Springerplus 2016, 5(1):1051.

34. Safatli DA, Gunther A, Schlattmann P, Schwarz F, Kalff R, Ewald C: Predictors of 30-day mortality in patients with spontaneous primary intracerebral hemorrhage. Surg Neurol Int 2016, 7(Suppl 18):S510-517.

35. Sembill JA, Gerner ST, Volbers B, Bobinger T, Lucking H, Kloska SP, Schwab S, Huttner HB, Kuramatsu JB: Severity assessment in maximally treated ICH patients: The max-ICH score. Neurology 2017, 89(5):423-431.

36. Gupta VP, Garton ALA, Sisti JA, Christophe BR, Lord AS, Lewis AK, Frey HP, Claassen J, Connolly ES, Jr.: Prognosticating Functional Outcome After Intracerebral Hemorrhage: The ICHOP Score. World Neurosurg 2017, 101:577-583.

37. DeLong ER, DeLong DM, Clarke-Pearson DL: Comparing the areas under two or more correlated receiver operating characteristic curves: a nonparametric approach. Biometrics 1988, 44(3):837-845.

38. Geurts M, Macleod MR, van Thiel GJ, van Gijn J, Kappelle LJ, van der Worp HB: End-of-life decisions in patients with severe acute brain injury. Lancet Neurol 2014, 13(5):515-524.

39. Morgenstern LB, Zahuranec DB, Sanchez BN, Becker KJ, Geraghty M, Hughes R, Norris G, Hemphill JC, 3rd: Full medical support for intracerebral hemorrhage. Neurology 2015, 84(17):1739-1744.

40. Anderson CS, Heeley E, Huang Y, Wang J, Stapf C, Delcourt C, Lindley R, Robinson T, Lavados P, Neal B et al: Rapid bloodpressure lowering in patients with acute intracerebral hemorrhage. N Engl J Med 2013, 368(25):2355-2365.

41. Qureshi Al, Palesch YY, Barsan WG, Hanley DF, Hsu CY, Martin RL, Moy CS, Silbergleit R, Steiner T, Suarez JI et al: Intensive Blood-Pressure Lowering in Patients with Acute Cerebral Hemorrhage. N Engl J Med 2016, 375(11):1033-1043.

42. Mendelow AD, Gregson BA, Fernandes HM, Murray GD, Teasdale GM, Hope DT, Karimi A, Shaw MD, Barer DH, investigators $S$ : Early surgery versus initial conservative treatment in patients with spontaneous supratentorial 
intracerebral haematomas in the International Surgical Trial in Intracerebral Haemorrhage (STICH): a randomised trial. Lancet 2005, 365(9457):387-397.

43. Mendelow AD, Gregson BA, Rowan EN, Murray GD, Gholkar A, Mitchell PM, Investigators SI: Early surgery versus initial conservative treatment in patients with spontaneous supratentorial lobar intracerebral haematomas (STICH II): a randomised trial. Lancet 2013, 382(9890):397-408.

44. Hanley DF, Thompson RE, Rosenblum M, Yenokyan G, Lane K, McBee N, Mayo SW, Bistran-Hall AJ, Gandhi D, Mould WA et al: Efficacy and safety of minimally invasive surgery with thrombolysis in intracerebral haemorrhage evacuation (MISTIE III): a randomised, controlled, open-label, blinded endpoint phase 3 trial. Lancet 2019, 393(10175):1021-1032.

45. Sprigg N, Flaherty K, Appleton JP, Al-Shahi Salman R, Bereczki D, Beridze M, Christensen H, Ciccone A, Collins R, Czlonkowska A et al: Tranexamic acid for hyperacute primary IntraCerebral Haemorrhage (TICH-2): an international randomised, placebo-controlled, phase 3 superiority trial. Lancet 2018, 391(10135):2107-2115.

46. Selim M, Foster LD, Moy CS, Xi G, Hill MD, Morgenstern LB, Greenberg SM, James ML, Singh V, Clark WM et al: Deferoxamine mesylate in patients with intracerebral haemorrhage (i-DEF): a multicentre, randomised, placebocontrolled, double-blind phase 2 trial. Lancet Neurol 2019, 18(5):428-438.

47. Collins FS, Varmus H: A new initiative on precision medicine. N Engl J Med 2015, 372(9):793-795.

\section{Figures}




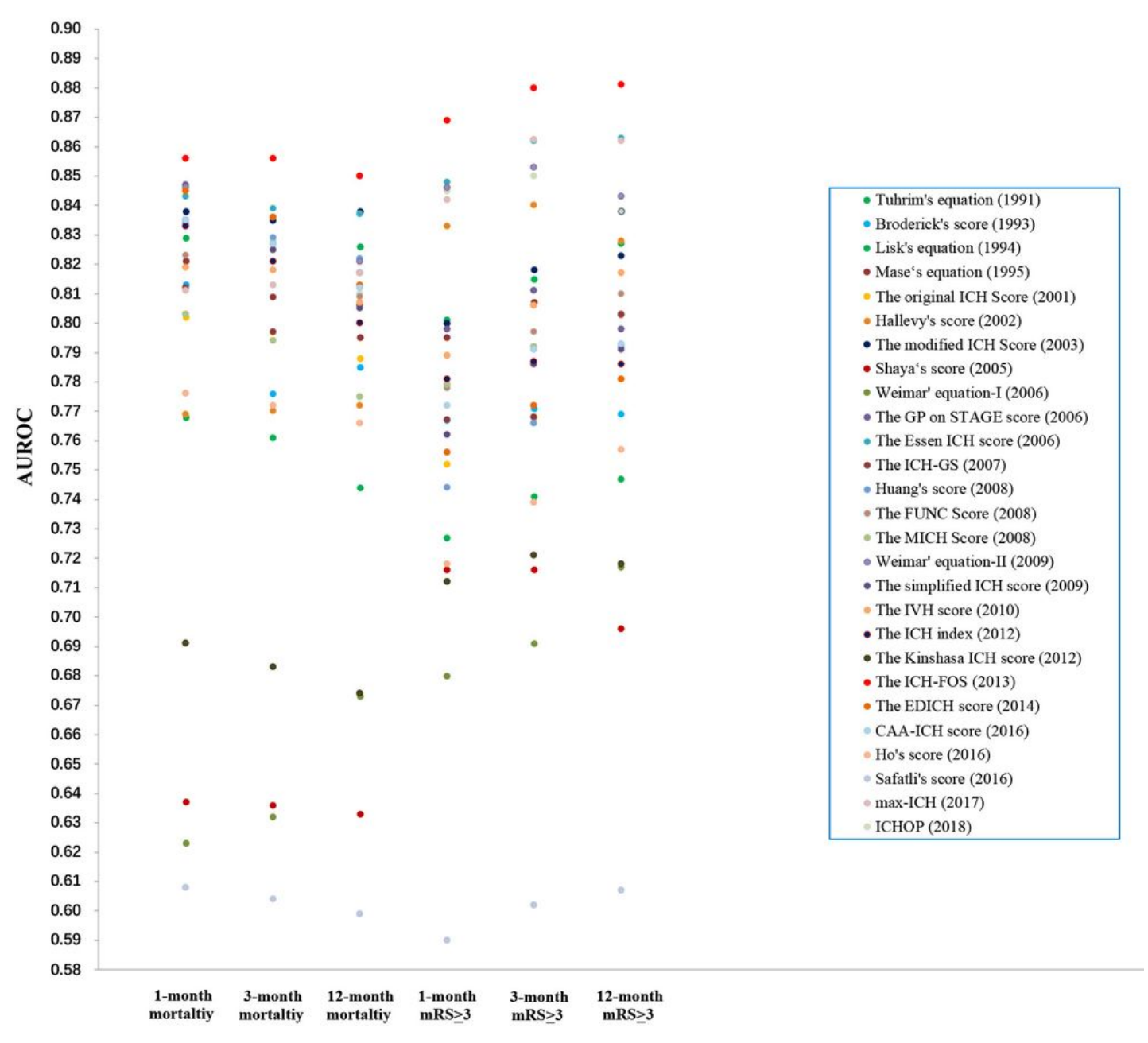

\section{Figure 1}

Predictive performance of $\mathrm{ICH}$ models with regard to mortality and poor functional outcome at 1-month, 3-month and 1-year after onset $(n=1575)$ 

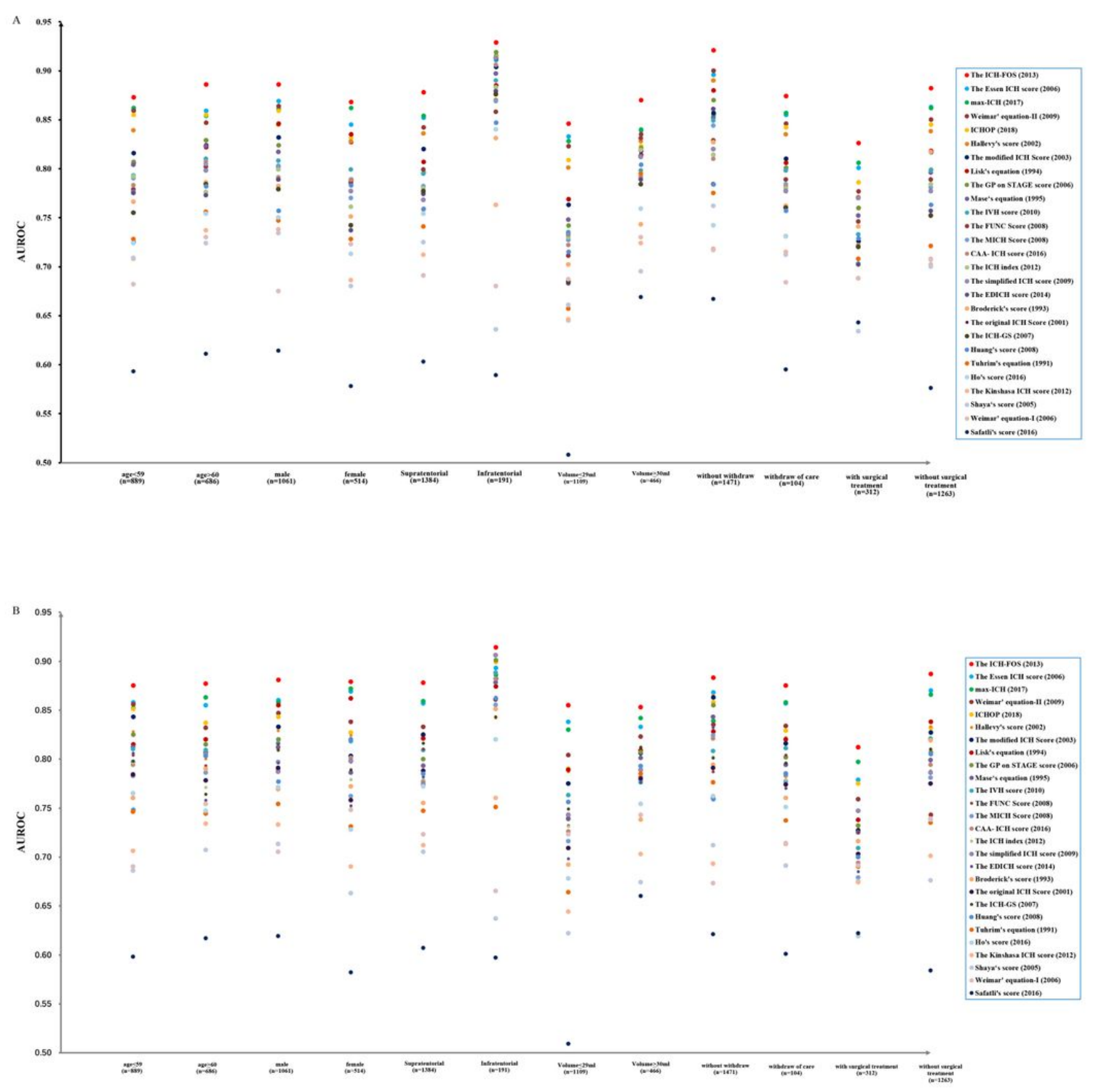

Figure 2

Sensitivity analysis of ICH models with regard to poor functional outcome (mRS $\geq 3$ ) at 3-month and 1-year after onset $(n=1575)$

\section{Supplementary Files}

This is a list of supplementary files associated with this preprint. Click to download.

- supplementarytablesandfigures.pdf 